Vol. 63 (2001) [123-131]

\title{
NONUNIQUENESS AND WELLPOSEDNESS OF ABSTRACT CAUCHY PROBLEMS IN A FRÉCHET SPACE
}

\author{
Peer Christian Kunstmann
}

Suppose that $A$ is a closed linear operator in a Fréchet space $X$. We show that there always is a maximal subspace $Z$ containing all $x \in X$ for which the abstract Cauchy problem has a mild solution, which is a Fréchet space for a stronger topology. The space $Z$ is isomorphic to a quotient of a Fréchet space $F$, and the part $A_{Z}$ of $A$ in $Z$ is similar to the quotient of a closed linear operator $B$ on $F$ for which the abstract Cauchy problem is well-posed. If mild solutions of the Cauchy problem for $A$ in $X$ are unique it is not necessary to pass to a quotient, and we reobtain a result due to R. deLaubenfels.

Moreover, we obtain a continuous selection operator for mild solutions of the inhomogeneous equation.

\section{INTRODUCTION}

Let $X$ be a Fréchet space and let $A$ be a closed linear operator in $X$. We shall be concerned with solutions of abstract Cauchy problems

$$
\begin{array}{lll}
u^{\prime}(t)=A u(t), & (t \geqslant 0), & u(0)=x \\
u^{\prime}(t)=A u(t)+f(t), & (t \geqslant 0), & u(0)=x,
\end{array}
$$

where $x \in X$ and the continuous function $f:[0, \infty) \rightarrow X$ are given.

It is well-known that, if $A$ is the generator of a $C_{0}$-semigroup $T=\left(T_{t}\right)_{t \geqslant 0}$ in $X$, then one gets mild solutions of (1) and (2) by $u(t)=T_{t} x, t \geqslant 0$, and $u(t)=T_{t} x+T * f(t)=$ $T_{t} x+\int_{0}^{t} T_{t-s} f(s) d s, t \geqslant 0$. It is also well-known that $A$ generates a $C_{0}$-semigroup if and only if (1) is well-posed, that is, there exists a unique mild solution of (1) for any $x \in X$. In the general case, however, there might be no nontrivial solution to (1), and, if solutions exist, they need not be unique.

Nevertheless, there are - under different additional assumptions - some results on "automatic well-posedness". If $X$ is a Banach space, Kantorowitz [6] has constructed a maximal subspace $H$ of $X$ (the Hille-Yosida space) which is a Banach space for a stronger topology $(H \hookrightarrow X)$ and on which the part $A_{H}$ of $A$ in $H$ generates a $C_{0}$-semigroup of linear

Received 12th April, 2000

Copyright Clearance Centre, Inc. Serial-fee code: 0004-9727/01 \$A2.00+0.00. 
contractions. Recall that the part $A_{B}$ of $A$ in $H$ is given by $x \in D\left(A_{H}\right)$ and $A_{H} x=y$ if and only if $x \in D(A) \cap H, y \in H$ and $A x=y$. He assumed that $(0, \infty) \subset \rho(A)$ where $\rho(A)$ denotes the resolvent set of $A$. Recall that if we assume in addition to $(0, \infty) \subset \rho(A)$ the well-known condition of Lyubich [8] on the growth of the resolvent $R(\lambda, A)$ as $\lambda \rightarrow \infty$, then solutions of (1) are unique in $X$.

Assuming the uniqueness of solutions of $(1)$ in $X$, deLaubenfels $[3,2,4]$ has constructed a maximal subspace $Z \hookrightarrow X$ (the solution space of $A$ ) on which the part $A_{Z}$ of $A$ in $Z$ generates a $C_{0}$-semigroup. In general this space is a Fréchet space even if the original space $X$ is a Banach space. Using the semigroup, one gets by the variation of constants formula unique (mild) solutions of (2) for $f \in C([0, \infty), Z), x \in Z$.

Recently, Herzog and Lemmert [5] used what they called nonlinear fundamental systems for continuous linear operators $A$ in a Fréchet space $X$ under the assumption that (1) is solvable on $[0, T]$ for any $x \in X$, and they used them to get solutions of (2) where $f \in C([0, T], X), x \in X$. A nonlinear fundamental system can be regarded as a substitute for a strongly continuous semigroup generated by $A$ since solutions of (2) are obtained in [5] by a variations of constants formula.

In this paper we consider solution spaces and solutions of (2) for arbitrary closed linear operators $A$. Our results are a "non-uniqueness analogue" to the construction of the solution space $Z$ in [3], and they shed some light on the role the uniqueness assumption plays in the construction.

It is easy to see that, even if an operator $A$ is well-posed in $X$ and $Y$ is an $A$-invariant subspace of $X$, one might lose existence of mild solutions of the abstract Cauchy problem for the part $A_{Y}$ of $A$ in $Y$, and one might lose uniqueness of mild solutions for the quotient operator $[A]_{X / Y}$ in the quotient $X / Y$, see Section 2 .

Our main result shows that this is as bad as it can get in the general situation: a linear operator $A$ for which solutions of (1) are not unique does not behave as badly as one might think, there always is a subspace $Z$ of $X$ which is a Fréchet space for a stronger topology and which is a quotient space of a Fréchet space on which a corresponding operator is well-posed. Precisely we shall show the following result.

TheOREM 1. Let $A$ be a closed linear operator in a Fréchet space $X$. Then there is a subspace $Z$ of $X$ which is a Fréchet space for a stronger topology such that $Z$ is a quotient of a Fréchet space $F$ and the part $A_{Z}$ of $A$ in $Z$ is the quotient of a closed linear operator $B$ in $F$ for which the abstract Cauchy problem is well-posed. The subspace $Z$ is maximal in the sense that it contains all $x \in X$ for which there is mild solution of (1).

Moreover, there is a continuous function $T: Z \times C([0, \infty), Z) \rightarrow C([0, \infty), Z)$ such that $T(x, f)$ is a mild solution of (2) for all $x \in Z, f \in C([0, \infty), Z)$.

The last statement allows us to use fixed point arguments to treat semilinear equations. For a situation were this has been done for a compact semilinearity we refer to $[5]$. 
The paper is organised as follows. In Section 2 we discuss well-posedness for the abstract Cauchy problem in subspaces and quotient spaces, and in Section 3 we prove Theorem 1. In Section 4 we illustrate our result by considering the heat equation in spaces of entire functions.

The author thanks G. Herzog and R. Lemmert for the inspiration for this work and for a copy of their preprint [5].

\section{WELL-POSEDNESS IN SUBSPACES AND QUOTIENT SPACES}

Suppose that $A$ is a closed linear operator in a Fréchet space $X$ which generates a $C_{0}$-semigroup of continuous linear operators $\left(T_{t}\right)_{t \geqslant 0}$. Let $Y$ be a closed linear subspace of $X$.

It is easy to see and well-known that if $Y$ is invariant under each $T_{t}$, then the restricted operators $\left(\left.T_{t}\right|_{Y}\right)$ and the quotient operators $\left(\left[T_{t}\right]_{X / Y}\right)$ are again $C_{0}$-semigroups in $Y$ and $X / Y$, respectively, with generators $A_{Y}$ (part of $A$ in $Y$ ) and $[A]_{X / Y}$, respectively. Here $[A]_{X / Y}$ means the quotient of $A$ in $X / Y$, that is, $[A]_{X / Y}:=\{([x],[y]):(x, y) \in A\}$.

If $X$ is Banach space then $Y$ is invariant under each $T_{t}$ if and only if $Y$ is invariant under the resolvents $(\lambda-A)^{-1}$ of $A$ for $\lambda$ large. This is because, for $\lambda$ large, $(\lambda-A)^{-1}$ is obtained by a Laplace transform from the semigroup, and, conversely, the semigroup operators $T_{t}$ may be obtained as a strong limit of the sequence $\left((n / t)^{n}(n / t-A)^{-n}\right)$. The equivalence no longer holds in a general Fréchet space since the resolvent set $\rho(A)$ of $A$ may be empty.

If the subspace $Y$ is $A$-invariant, that is, $A y \in Y$ for any $y \in D(A) \cap Y$, then the operator $[A]_{X / Y}$ is still a closed linear operator in $X / Y$. If $Y$ is invariant under the semigroup then $Y$ is $A$-invariant, hence $A$-invariance is a weaker assumption. If $A$ is a bounded operator and $X$ is a Banach space then $A$-invariance implies invariance under the semigroup generated by $A$. In general this is not the case as the following example shows.

EXAMPLE 2. Let $X$ denote the space of all bounded uniformly continuous scalar functions $f$ on $[0, \infty)$ that satisfy $f(0)=0$. Let $A:=-d / d x$ with $D(A):=\left\{f \in X: f^{\prime} \in X\right\}$. The operator $A$ generates the $C_{0}$-semigroup $\left(T_{t}\right)$ given by

$$
T_{t} f(x)=f(x-t) \text { if } x \geqslant t,=0 \text { otherwise } .
$$

Let $Y$ be the linear span of the function $x \mapsto \sin x$. Since $D(A) \cap Y=\{0\}$, the subspace $Y$ is $A$-invariant. But for any $f \in Y \backslash\{0\}$ and any $t>0$ the function $T_{t} f$ does not belong to $Y$. Hence the Cauchy problem for $A_{Y}$ in $Y$ has no nontrivial solution, and for any $f \in Y \backslash\{0\}$, the function $t \mapsto T_{t} f+Y$ is a nontrivial mild solution of $u^{\prime}=[A]_{X / Y} u$, $u(0)=0$, in $X / Y$. 


\section{The MAXIMAL SOlUtion SPACE}

We assume that $A$ is a closed linear operator in a Fréchet space $X$ with domain $D(A)$. We consider mild solutions of (1) and (2), that is, continuous solutions of

$$
\begin{aligned}
& u(t)=A\left(\int_{0}^{t} u(s) d s\right)+x \quad(t \geqslant 0), \\
& u(t) \doteq A\left(\int_{0}^{t} u(s) d s\right)+\int_{0}^{t} f(s) d s+x \quad(t \geqslant 0) .
\end{aligned}
$$

From now on we shall denote by $1 * u$ the function $t \mapsto \int_{0}^{t} u(s) d s$ on $[0, \infty)$ where $u$ is a given function on $[0, \infty)$.

Let $E$ denote the space $C([0, \infty), X)$. Then $E$ is a Fréchet space for the family of seminorms $p_{k, n}(f):=\sup _{s \in[0, k]} q_{n}(f(s)), k, n \in \mathbb{N}$, where $\left(q_{n}\right)_{n \in \mathbb{N}}$ is a defining family of seminorms for the topology of $X$. The space

$$
F:=\{u \in E: \forall t \geqslant 0,1 * u(t) \in D(A), u(t)=A(1 * u(t))+u(0)\}
$$

is a closed linear subspace of $E$. Indeed, $u_{n} \rightarrow u$ in $E$ for a sequence $\left(u_{n}\right)$ in $F$ means $q_{m}\left(u_{n}-u\right) \rightarrow 0$ uniformly on compact intervals for any $m \in \mathbb{N}$. This implies $1 * u_{n}(t) \rightarrow$ $1 * u(t)$ and $u_{n}(0) \rightarrow u(0)$. On the other hand, it also gives $A\left(1 * u_{n}(t)\right)=u_{n}(t)-u_{n}(0) \rightarrow$ $u(t)-u(0)$ which implies $u \in F$ by the closedness of $A$. Clearly, $F$ is the space of all continuous solutions of (3).

For each $t \geqslant 0$, we define the linear continuous map $\Phi_{t}: F \rightarrow X, u \mapsto u(t)$, and let $Z$ denote the range $\Phi_{0}(F)$ of $\Phi_{0}$. We equip $Z$ with the topology of the quotient space $F /$ ker $\Phi_{0}$ induced by the factorisation $\widehat{\Phi}_{0}: F / \operatorname{ker} \Phi_{0} \rightarrow Z$ of $\Phi_{0}$. Then $Z$ is a Fréchet space and $\Phi_{0}: F \rightarrow Z$ is continuous and onto. By [1, Chapter 4, Proposition 12], there exists a continuous right inverse $Q: Z \rightarrow F$, that is, $Q$ satisfies $\Phi_{0} \circ Q=\mathrm{Id}_{z}$. Of course, if $\Phi_{0}$ is injective (the case of uniqueness) then $F$ is isomorphic to $Z$ and $Q=\Phi_{0}^{-1}$ is linear and continuous. If $\Phi_{0}$ is not injective, $Q$ can be chosen to be linear if and only if $\operatorname{ker} \Phi_{0}$ is a complemented subspace of $F$, that is, if and only if there is a continuous linear projection $p: F \rightarrow \operatorname{ker} \Phi_{0}$, the relation being $\operatorname{Id}_{z}-p=Q \circ \Phi_{0}$. Hence in general $Q$ is not linear. In any case, however, $Q$ can be interpreted as a selection of solutions to (1) which depend continuously (in the topology of $Z$ ) on the initial value $x$. The following proposition collects the properties of this construction and proves the first part of Theorem 1.

PROPOSITION 3.

(i) For each $t \geqslant 0$ the map $\Phi_{t}: F \rightarrow Z$ is linear and continuous;

(ii) For each $t \geqslant 0$ the map $T_{t}: F \rightarrow F, u \mapsto u(\cdot+t)$ is linear and continuous;

(iii) The family $\left(T_{t}\right)_{t \geqslant 0}$ defines a $C_{0}$-semigroup in $F$ whose generator $B$ is given by $B u=u^{\prime}$ on $D(B):=\left\{u \in C^{1}([0, \infty), X): u, u^{\prime} \in F\right\}$; here $u^{\prime}$ denotes the derivative taken pointwise in $X$. 
(iv) For each $t \geqslant 0$ we have $\Phi_{t} B \subset A_{Z} \Phi_{t}$, the space ker $\Phi_{0}$ is $B$-invariant and $A_{Z}=\widehat{\Phi}_{0}[B]_{F / \operatorname{ker} \Phi_{0}} \widehat{\Phi}_{0}^{-1}$.

Proof: (i) follows from (ii) by $\Phi_{t}=\Phi_{0} T_{t}$, so we start with the proof of (ii). If $u \in F$ and $t \geqslant 0$ then, for any $s \geqslant 0$, we have

$$
\begin{aligned}
u(s+t)-u(t) & =A(1 * u(s+t)-1 * u(t))+u(0)-u(0) \\
& =A\left(\int_{t}^{s+t} u(r) d r\right) \\
& =A(1 * u(\cdot+t))(s)
\end{aligned}
$$

which implies $T_{t} u \in F$. The continuity of $T_{t}$ is clear since $p_{k, n}\left(T_{t} u\right) \leqslant p_{m, n}(u)$ for any $m \geqslant k+t, n \in \mathbb{N}$, and $u \in F$.

Any $u \in F$ is uniformly continuous on compact intervals which implies $T_{t} u \rightarrow u$ in $F$ as $t \rightarrow 0$, that is, $\left(T_{t}\right)$ is a strongly continuous semigroup in $F$. Let $B$ denote its generator. If $u \in D(B)$ and $B u=v$ then $\left(T_{t} u-u\right) / t$ converges to $v$ in $F$ as $t \rightarrow 0$ hence also pointwise in $X$. This gives $v=u^{\prime}$. On the other hand, if $u \in C^{1}([0, \infty), X)$ with $u, u^{\prime} \in F$ then the uniform continuity of $u^{\prime}$ on compact intervals gives $\left(T_{t} u-u\right) / t \rightarrow u^{\prime}$ in $f$ as $t \rightarrow 0$. So (iii) is proved.

(iv) Let $t \geqslant 0$ and $u \in D(B)$. Then, for $s \geqslant 0$,

$$
\frac{1}{s}(u(t+s)-u(t))=A\left(\int_{t}^{s+t} \frac{u(r)}{s} d r\right)
$$

The integral on the right hand side tends to $u(t)$ in $X$ as $s \rightarrow 0$ and, since $u$ is differentiable in $t$, the left hand side tends to $u^{\prime}(t)$ in $X$ as $s \rightarrow 0$.

By the closedness of $A$ we get $u(t) \in D(A)$ and $u^{\prime}(t)=A u(t)$, that is, $\Phi_{t} B u=A \Phi_{t} u$, which means $\Phi_{t} B \subset A_{Z} \Phi_{t}$ since, by (i), $\Phi_{t} u, \Phi_{t} B u \in Z$. In particular we have $\Phi_{0} B \subset$ $A_{z} \Phi_{0}$.

If $u \in D(B) \cap \operatorname{ker} \Phi_{0}$, that is, $u, u^{\prime} \in F$ and $u(0)=0$, then

$$
u^{\prime}(0)=(B u)(0)=\Phi_{0} B u=A_{Z} \Phi_{0} u=A u(0)=0,
$$

that is, $B u=u^{\prime} \in \operatorname{ker} \Phi_{0}$. Hence $\operatorname{ker} \Phi_{0}$ is $B$-invariant.

Recall the definition of the quotient operator and note that $[B]:=[B]_{F / \text { ker } \Phi_{0}}=$ $\left\{\left([u],\left[u^{\prime}\right]\right): u \in D(B)\right\}$. Now let $u \in D(B)$. Then, by the above, $\widehat{\Phi}_{0}[B][u]=\Phi_{0} B u=$ $A_{Z} \Phi_{0} u$. Hence $\widehat{\Phi}_{0}[B] \subset A_{Z} \widehat{\Phi}_{0}$, which implies $\widehat{\Phi}_{0}[B] \widehat{\Phi}_{0}^{-1} \subset A_{Z}$. To prove the reverse inclusion let $x \in D\left(A_{Z}\right)$, that is, $x \in D(A) \cap Z$ and $A x \in Z$. Choose $v \in F$ such that $v(0)=A x$. Let $u:=1 * v+x$. Then $u(0)=x$ and for any $t \geqslant 0$ we have by the choice of $v$ :

$$
u^{\prime}(t)=v(t)=A(1 * v(t))+A x=A(1 * v(t)+x)=A u(t)
$$


Hence $u$ is a classical (in particular, a mild) solution for the initial value $x$, that is, $[u]=\widehat{\Phi}_{0}^{-1} x$. By the arguments used in the proof of (ii) we have $u \in D(B)$, which implies $x \in D\left(\widehat{\Phi}_{0}[B] \widehat{\Phi}_{0}^{-1}\right)$, and the proof of (iv) is complete.

The remaining part of Theorem 1 is proved in the following

Proposition 4. There is a continuous function $T: Z \times C([0, \infty), Z) \rightarrow$ $C([0, \infty), Z)$ such that $T(x, f)$ is a solution of (4) for all $x \in Z, f \in C([0, \infty), Z)$.

Proof: It is well-known that the map, $S:(u, g) \mapsto T_{(\cdot)} u+T * g$ is a continuous function $F \times C([0, \infty), F) \rightarrow C([0, \infty), F)$ such that $S(u, g)$ is a solution of

$$
v(t)=B(1 * v)(t)+(1 * g)(t)+u \quad(t \geqslant 0) .
$$

Define $T$ by $T(x, f):=\Phi_{0} S(Q x, Q \circ f)$. By the continuity of $Q, S$ and $\Phi_{0}$, the function $T$ is continuous from $Z \times C([0, \infty), Z)$ to $C([0, \infty), Z)$. By applying $\Phi_{0}$ to $(5)$ and using Proposition 3 (iv), we see that $T(x, f)$ is a continuous solution of (4).

Remark 5. (i) The solution $T(0, f)$ in the proof of Proposition 4 is given by

$$
\begin{aligned}
u(t) & =\Phi_{0} \int_{0}^{t} T_{t-s} Q(f(s)) d s \\
& =\int_{0}^{t} \Phi_{t-s}(Q(f(s))) d s \\
& =\int_{0}^{t}(Q(f(s)))(t-s) d s .
\end{aligned}
$$

The last expression appeared in [5] for continuous operators $A$ in a Fréchet space $X$ for which $Z=X$. It justifies the term nonlinear fundamental system used by $\mathrm{G}$. Herzog and $\mathrm{R}$. Lemmert for the (in general nonlinear) selection operator $Q$. Notice that in [5] any mild solution is a classical solution due to the continuity of $A$.

(ii) Solutions of (1) are unique if and only if $\Phi_{0}$ is injective. In this case, $\Phi_{0}: F \rightarrow Z$ is an isomorphism, $Q=\Phi_{0}^{-1}$ and $U_{t}:=\Phi_{0} T_{t} \Phi_{0}^{-1}=\Phi_{t} \circ Q$ defines, by similarity, a $C_{0^{-}}$ semigroup $\left(U_{t}\right)_{t \geqslant 0}$ in $Z$ whose generator can be shown to be the part $A_{Z}$ of $A$ in $Z$ given by $A_{Z} x=A x$ on $D\left(A_{Z}\right):=\{x \in D(A) \cap Z: A x \in Z\}$. This result is due to deLaubenfels $[3,4]$. The arguments used in the construction of $Z$ and the semigroup generated by the part of $A$ in $Z$ are similar to ours but are carried out directly in $Z$ rather than in $F$.

(iii) Notice that the operators $Q_{t}:=\Phi_{t} \circ Q$ are in general nonlinear, and it is not clear if $Q$ can be chosen in such a way that $Q_{t} \circ Q_{s}=Q_{s+t}$ for all $s, t \geqslant 0$, in which case $\left(Q_{t}\right)_{t \geqslant 0}$ would be a strongly continuous semigroup of continuous nonlinear operators in the Fréchet space $Z$.

It will in general be impossible to construct the solution space $Z$ for a given operator $A$. The following corollary is easier to apply since it only requires finding sufficiently many initial values for which (1) has a (mild) solution. 
COROLlary 6 . Let $W \hookrightarrow X$ be an ultrabornological topological vector space such that (1) has a mild solution for any $x \in W$ in the sense of (3). Then (2) has a mild solution for any $f \in C([0, \infty), W)$ in the sense of (4).

Proof: We have $W \subset Z$, and the inclusion is closed. By the closed graph theorem $[7$, p.57, (2)], and $[7$, p.55, (4)], we get $W \hookrightarrow Z$. Hence $C([0, \infty), W) \hookrightarrow C([0, \infty), Z)$, and Proposition 4 gives the assertion.

\section{THE HEAT EQUATION IN SPACES OF ENTIRE FUNCTIONS}

In this section we consider the one-dimensional heat equation

$$
u_{t}=u_{x x}, \quad t \geqslant 0, x \in \mathbf{R}, \quad u(0, x)=f(x), \quad x \in \mathbf{R}
$$

in spaces of entire functions. For simplicity we only consider even functions

$$
f(x)=\sum_{k=0}^{\infty} \frac{c_{k}}{(2 k) !} x^{2 k}, \quad x \in \mathbb{C} .
$$

By the Cauchy-Hadamard formula, (7) defines an entire function if and only if the sequence $\left(c_{k}\right)$ satisfies

$$
\sup _{k \geqslant 0} \frac{h^{k}\left|c_{k}\right|}{(2 k) !}<\infty, \quad h>0
$$

The space of all these functions is clearly a Fréchet space which we denote by $E$. More generally, we consider function spaces $E_{\omega} \subset E$ where $\omega:[0, \infty) \rightarrow[0, \infty)$ is a nondecreasing continuous function satisfying
(i) $\omega(2 r)=O(\omega(r)) \quad(r \rightarrow \infty)$,
(ii) $\sqrt{r}=O(\omega(r)) \quad(r \rightarrow \infty)$,
(iii) $\varphi: t \mapsto \omega\left(e^{t}\right)$ is convex.

We denote by $\varphi^{*}$ the convex conjugated function $\varphi^{*}(s):=\sup _{t \geqslant 0}(s t-\varphi(t))$ and define $E_{\omega}$ to be the space of all functions $f$ of the form (7) such that $\left(c_{k}\right) \in \Lambda_{\omega}$ where

$$
\Lambda_{\omega}:=\left\{\left(c_{k}\right): \forall m \in \mathbb{N}, q_{\omega, m}\left(c_{k}\right):=\sup _{k}\left|c_{k}\right| e^{-m \varphi^{*}(k / m)}<\infty\right\}
$$

Observe that $E_{\omega}=E$ for $\omega(r)=\sqrt{r}$ by Stirling's formula and (8), and that (ii) implies that $E_{\omega} \subset E$. Clearly $\Lambda_{\omega}$ is a Fréchet space for the family of norms $\left(q_{\omega, m}\right)_{m \in \mathbb{N}}$ and we consider the topology on $E_{\omega}$ induced by the linear bijection $f \mapsto\left(c_{k}\right)$.

Then a mild solution to the heat equation in $E_{\omega}$ corresponds to a continuous function $g=\left(g_{k}\right):[0, \infty) \rightarrow \Lambda_{\omega}$ satisfying the infinite system

$$
g_{k}^{\prime}(t)=g_{k+1}(t), \quad t \geqslant 0 ; \quad g_{k}(0)=c_{k} .
$$


By induction we see that $g_{k}=g_{0}^{(k)}$ for all $k \in \mathbb{N}_{0}$. Hence a solution corresponds to an element of the space

$$
\mathcal{E}_{\omega}^{+}:=\left\{g \in C^{\infty}[0, \infty): \forall m, n \in \mathbb{N}, p_{\omega, m, n}^{+}(g):=\sup _{0 \leqslant t \leqslant n} \sup _{k \in \mathbb{N}_{0}}\left|g^{(k)}(t)\right| e^{-m \varphi^{*}(k / m)}<\infty\right\} .
$$

The family of seminorms $\left(p_{\omega, m, n}^{+}\right)_{m, n}$ turns $\mathcal{E}_{\omega}^{+}$into a Fréchet space. We also define the space $\mathcal{E}_{\omega}$ of all functions $g \in C^{\infty}(\mathbb{R})$ such that $p_{\omega, m, n}(g)<\infty$ where $p_{\omega, m, n}$ is defined as $p_{\omega, m, n}^{+}$with $\sup _{0 \leqslant t \leqslant n}$ replaced by sup. Then solutions of the heat equation in $E_{\omega}$ are unique if and only if $g \in \mathcal{E}_{\omega}^{+}$and $g^{(k)}(0)=0$ for all $k \in \mathbb{N}_{0}$ imply $g=0$, that is, if and only if $\mathcal{E}_{\omega}^{+}$ is quasi-analytic, which is known to be the case if and only if $\omega$ satisfies

$$
\int_{1}^{\infty} \frac{\omega(r)}{r^{2}} d r=\infty
$$

(This is a version of the Denjoy-Carleman Theorem [9, Theorem 19.11]: letting $M_{k}=$ $\exp \left(m \varphi^{*}(k / m)\right)$ we obtain by $\varphi=\varphi^{* *}$ that $\log q(x) \sim m \omega(x)$; see also [10].) Hence we concentrate on the case $\int_{1}^{\infty} \omega(r) r^{-2} d r<\infty$. In this case the heat equation has a mild solution for all initial values $f \in E_{\omega}$ if and only if the map $\delta_{\omega}^{+}: \mathcal{E}_{\omega}^{+} \rightarrow \Lambda_{\omega}, g \mapsto\left(g^{(k)}(0)\right)$ is surjective. Since it is easy to see that $\delta_{\omega}^{+}$is surjective if and only if $\delta_{\omega}: \mathcal{E}_{\omega} \rightarrow \Lambda_{\omega}$ is surjective we have by $[10$, Theorem 3.10$]$ that this is the case if and only if

$$
\int_{0}^{\infty} \frac{\omega(y r)}{1+r^{2}} d r \leqslant C \omega(y)+C, \quad y \geqslant 0 .
$$

This condition holds for $\omega(r)=r^{\alpha}, 1 / 2 \leqslant \alpha<1$ (actually also for $0<\alpha<1 / 2$ but (ii) requires $\alpha \geqslant 1 / 2)$ whereas it does not hold for $\omega(r)=r(\log r)^{-\beta}$ (see [10, Example 1.8]).

Thus we have the following: if (10) holds then the solution space is $E_{\omega}$; if (10) does not hold then the solution space is $\operatorname{im} \delta_{\omega}^{+} \neq E_{\omega}$. In either case the solution space is isomorphic to the quotient space $\mathcal{E}_{\omega}^{+} / \operatorname{ker} \delta_{\omega}^{+}$. If $h:[0, \infty) \rightarrow \operatorname{im} \delta_{\omega}^{+}$is a continuous function then there exists a mild solution $u:[0, \infty) \rightarrow E_{\omega}$ of the equation $u_{t}=u_{x x}+h(t, x), t \geqslant 0$, $u(0)=0$.

\section{REFERENCES}

[1] N. Bourbaki, Topological vector spaces (Springer-Verlag, Berlin, Heidelberg, New York, 1987).

[2] R. deLaubenfels, ' $C$-semigroups and strongly continuous semigroups', Israel J. Math. 81 (1993), 227-255.

[3] R. deLaubenfels, 'Automatic well-posedness with the abstract Cauchy problem on a Frechet space', J. London Math. Soc. Ser. 248 (1993), 526-536.

[4] R. deLaubenfels, Existence families, functional calculi and evolution equations, Lecture Notes Maths 1570 (Springer-Verlag, Berlin, Heidelberg, New York, 1994).

[5] G. Herzog and R. Lemmert, 'Nonlinear fundamental systems for linear differential equations in Fréchet spaces', Demonstratio Math. 33 (2000), 313-318. 
[6] S. Kantorovitz, 'The Hille-Yosida space of an arbitrary operator', J. Math. Anal. Appl. 136 (1988), 107-111.

[7] G. Köthe, Topological vector spaces II (Springer-Verlag, New York, Heidelberg, Berlin, 1979).

[8] Yu.I. Lyubich, 'Conditions for the uniqueness of the solution to Cauchy's abstract problem', Soviet Math. Dokl. 1 (1960), 110-113.

[9] W. Rudin, Real and complex analysis, Series in Higher Mathematics (McGraw-Hill, New York, Dusseidorf, Johannesburg, 1987).

[10] R. Meise and B.A. Taylor, 'Whitney's extension theorem in spaces of ultradifferentiable functions of Beurling type', Ark. Mat. 26 (1988), 265-287.

Mathematisches Institut I

Universität Karlsruhe

Englerstr. 2, 76128 Karlsruhe

Germany

e-mail: peer.kunstmann@math.uni-karlsrube.de 